\title{
Artigo Original / Original Paper \\ Navicula (Naviculaceae) no perifíton de riachos e novas ocorrências para o estado de Goiás
}

\author{
Navicula (Naviculaceae) in the periphyton of streams and new occurrences for Goiás state
}

\author{
Daiane Trevisan Ruwer ${ }^{1,3,4}$, Alline Alves França ${ }^{2} \&$ Sirlene Aparecida Felisberto ${ }^{2}$
}

\begin{abstract}
Resumo
Este trabalho teve como objetivo inventariar e descrever as espécies de Navicula encontradas no perifíton em riachos do estado de Goiás, e contribuir com novos registros para o estado com a identificação de novas características taxonômicas. Amostras foram coletadas em nove ambientes lóticos, dos quais o material perifítico foi removido de gramíneas e seixos submersos. Foram preparadas lâminas permanentes com o material oxidado para observação, identificação e descrição dos espécimes. Como resultado, foram identificadas 10 espécies, das quais quatro são novos registros para o estado: Navicula leptostriata, Navicula symmetrica, Navicula neomundana e Navicula parablis. Os táxons mais frequentes foram Navicula leptostriata e Navicula parablis.
\end{abstract}

Palavras-chave: Centro-Oeste do Brasil, Cerrado, diatomáceas, taxonomia.

\begin{abstract}
This study aimed to describe and inventory the species of Navicula present in the periphyton of the Goiás state streams, and to contribute to state records with the identification of new taxonomic characteristics. Samples were collected from nine lotic environments, where the periphytic material was removed from submerged Gramineae and stones. Afterwards, to facilitate the observation, identification, and description of specimens, slides were prepared from oxidized material. As a result, ten species were identified, four of which were newly recorded in the state: Navicula leptostriata, Navicula symmetrica, Navicula neomundana, Jarlman \& and Navicula parablis. The most common taxon identified in this study was Navicula leptostriata, followed by Navicula parablis.
\end{abstract}

Key words: Central-western Brazil, Cerrado, diatoms, taxonomy.

\section{Introdução}

A flora de diatomáceas (Bacillariophyta) constitui uma parcela significativa na comunidade de algas perifíticas de ambientes lóticos (Bere \& Tundisi 2010; Patrick \& Reimer 1966). É de fundamental importância a correta delimitação e identificação dos táxons de diatomáceas para o diagnóstico e caracterização dos ambientes aquáticos. A relação entre diatomáceas e variáveis ambientais são robustas e quantificáveis fazendo destas indicadores adequados de condições ecológicas em sistemas lóticos (Bere \& Tundisi 2010; Pan et al. 1996; Oliveira et al. 2001).

Um dos grupos taxonômicos de diatomáceas que contribuem significamente para a riqueza em regiões tropicais é o gênero Navicula Bory (Leandrini et al. 2002; Rodrigues 1992; Wengrat et al. 2007). Navicula foi originalmente descrito em 1822 por Bory de St. Vincent, pertence à família Naviculaceae, e se distingue morfologicamente de outros gêneros dessa família por apresentar valvas em forma de barco ou fuso, variavelmente lanceoladas e com ápices de vários formatos

\footnotetext{
${ }^{1}$ Universidade Estadual de Maringá (UEM), Depto. Biologia Geral (DBI) e Núcleo de Pesquisa em Limnologia, Ictiologia e Aquicultura (Nupélia), Lab. Algas Perifíticas, Prog. Pós-graduação em Ecologia de Ambientes Aquáticos Continentais (PEA-UEM), 87020-900, Maringá, PR, Brasil.

${ }^{2}$ Universidade Federal de Goiás, Depto. Biologia Geral, Campus II, Inst. Ciências Biológicas, Prog. Pós-graduação em Biodiversidade Vegetal, CP 131, 74001970, Goiânia, GO, Brasil.

${ }^{3}$ ORCID: <https://orcid.org/0000-0002-5965-8793>

${ }^{4}$ Autor para correspondência: dai.ruwer@gmail.com
} 
(Round et al. 1990; Cox 1999; Lange-Bertalot 2001). Além disso, possuem estrias unisseriadas compostas de aréolas alongadas longitudinalmente, com sistema de rafe simples, costela acessória interna e dois plastídios marginais (Lange-Bertalot 2001). São espécies com indivíduos solitários ou coloniais formando tubos de mucilagem, nunca formando cadeias ou outras formas agregadas (Lange-Bertalot 2001).

Inicialmente, o gênero Navicula abrangeu muitos táxons de diatomáceas, tornando-o um conjunto heterogêneo de espécies que apresentavam apenas morfologia semelhante (Round et al. 1990; Cox 1999). A modificação da definição morfológica deste grupo heterogêneo começou antes mesmo de sua nova tipificação, com a proposição de novos subgêneros por Patrick (1959) e Hustedt (1959). Novos estudos taxonômicos e o surgimento das análises de microscopia eletrônica possibilitaram a proposição de novos gêneros, como por exemplo: Sellaphora D.G. Mann, Hippodonta Lange-Bertalot, Witkowski \& Metzeltin e Craticula Grunow (Mann 1989; Cox 1999; Torgan et al. 2010; Lange-Bertalot 2001). Esta reestruturação do gênero e a nova tipicação por Navicula tripunctata Bory, tornou esse grupo mais homogêneo taxonomicamente (Lange-Bertalot 2001). Apesar das mudanças significativas na sistemática do gênero Navicula, este ainda mantém uma grande riqueza de espécies em relação à outros gêneros (Kulikovskiy 2009).

Apesar da grande riqueza de espécies do gênero no Brasil, cerca de 63 espécies (Flora do Brasil 2020), e principalmente sua alta ocorrência em ambientes lóticos brasileiros (Leandrini et al. 2002; Rodrigues 1992; Wengrat et al. 2007), apenas 23 espécies de Navicula foram registradas para o estado de Goiás (Silva et al. 2011). Portanto, este trabalho visou realizar um levantamento de espécies de Navicula do perifíton de ambientes aquáticos lóticos. Como também teve como objetivo contribuir com o conhecimento taxonômico do gênero e com novos registros para o estado. Este estudo faz parte de um projeto maior sobre a diversidade de diatomáceas de ambientes aquáticos de Goiás, que abrange uma área de $340.111,783 \mathrm{~km}^{2}$ do Centro-Oeste brasileiro.

\section{Material e Métodos}

A região dos ambientes coletados encontrase no domínio fitogeográfico Cerrado, com clima tropical semi-úmido, onde nascem rios que abastecem cinco unidades hidrográficas brasileiras:
Amazônica, Tocantins-Araguaia, Paraguai, Paraná e São Francisco (PNRH 2006).

Foram amostrados nove ambientes lóticos (três córregos, cinco rios e uma cachoeira), os quais estão distribuídos em municípios nas regiões centro, nordeste, leste, norte e sudeste do estado de Goiás (Pirenópolis, Formosa, Alto Paraíso de Goiás, Alvorada do Norte, Flores de Goiás, Caldas Novas, Goiânia e Nova Veneza). Dentre os ambientes, dois estão localizados em regiões de preservação ambiental e são também utilizados em atividades recreativas: Corredeira dos Ingleses (P1) e Córrego Salto do Itiquira (P4). Os ambientes utilizados para atividades recreativas e localizados em propriedades particulares são: Córrego Pirenópolis, Cachoeira Pirenópolis Andorinha (P2 e P3). Os pontos que apresentam maior impacto antrópico e que são utilizados para lançamento de efluentes são: Córrego Santa Maria e Rio João Leite (P5 e P6). Os demais locais estão localizados próximos de propriedades com atividades pecuárias: Rio Pirapetinga, Rio após Nova Veneza e Ribeirão do Bragre (P7, P8 e P9) (Tab. 1).

As amostragens ocorreram nos anos de 2008, 2011 e 2012. Foram coletados pecíolos de gramíneas submersas da região marginal e/ ou seixos submersos. O material perifítico foi removido através da raspagem dos substratos com escova de cerdas e o auxílio de jatos d'água destilada. As amostras foram armazenadas e fixadas em solução Transeau (Bicudo \& Menezes 2017).

O material foi oxidado de acordo com a técnica Simonsen (1974) modificado por Moreira-Filho \& Valente-Moreira (1981). Lâminas permanentes, contento $1 \mathrm{~mL}$ da amostra, foram montadas com o meio de inclusão Naphrax. A análise qualitativa baseou-se em estudo populacional e foram realizadas em microscópio óptico binocular Zeiss Axioskop 40 com o sistema Axiovision em aumento de $1.000 \times$ para a captura de imagens. Para a análise das características ultraestruturais das espécimes foi utilizado o microscópio eletrônico de varredura Jeol JSM-6610 (MEV) do laboratório de microscopia de alta resolução - Universidade Federal de Goiás. O material analisado em MEV foi preparado de acordo com Ferrario et al. (1995). Esta preparação visa na montagem de lamínulas com alíquotas do material oxidado, coladas em suportes metálicos com tinta condutiva de carbono e metalizadas com ouro para observação.

A frequência de ocorrência de cada espécie foi realizado através da análise qualitativa das amostras. Os cálculos de frequência foram baseados 
em Guille (1970), segundo o qual os táxons com freqüências de até $10 \%$ são considerados raros, entre $10 \%$ e $50 \%$ são considerados comuns e mais de $50 \%$ foram considerados constantes ou bastante frequentes (Tab. 2). O sistema de classificação taxonomico utilizado foi o de Round et al. (1990). As amostras foram depositadas no acervo do Herbário da Universidade Federal de Goiás (Tab. 1).

\section{Resultados e Discussão}

Foram identificadas 10 espécies do gênero Navicula Bory (Tabs. 2 e 3) sendo quatro novos registros para o estado de Goiás: Navicula leptostriata Jørgensen. Navicula symmetrica Patrick, Navicula neomundana Lange-Bertalot, Jarlman \& van de Vijver e Navicula parablis Hohn \& Hellerman. Das quais as espécies $N$. leptostriata e Navicula parablis foram as mais frequentes do estudo (Tab. 2).

Divisão: Bacillariophyta

Classe Bacillariophyceae

Sub-Classe: Bacillariophycidae

Ordem: Naviculales Bessey

Sub-ordem: Naviculineae Hendey

Família: Naviculaceae Kützing Gênero: Navicula Bory

Tabela 1 - Dados da localização dos ambientes amostrados, de período de coleta, e número de herbário das amostras perifíticas do estado de Goiás utilizadas no estudo.

Table 1 - Data of the location of the sampled environments, the collection period, and the herbarium number of the periphytic samples from the state of Goiás used in the study.

\begin{tabular}{|c|c|c|c|c|c|c|}
\hline Ponto & Ambientes & Data & Localização & Região & Coordenadas & Herbário \\
\hline p1 & Corredeira dos Ingleses & $21 / 10 / 2011$ & $\begin{array}{l}\text { Alto Paraíso de } \\
\text { Goiás }\end{array}$ & Norte & $14^{\circ} 09^{\prime} \mathrm{S} / 47^{\circ} 50^{\prime} \mathrm{W}$ & UFG50331 \\
\hline $\mathrm{p} 2$ & Córrego Pirenópolis & $16 / 12 / 2011$ & Pirenópolis & Leste & $15^{\circ} 51^{\prime} \mathrm{S} / 48^{\circ} 55^{\prime} \mathrm{W}$ & UFG50326 \\
\hline p3 & $\begin{array}{l}\text { Cachoeira Pirenópolis } \\
\text { Andorinha }\end{array}$ & $16 / 12 / 2011$ & Pirenópolis & Leste & $15^{\circ} 50^{\prime} \mathrm{S} / 48^{\circ} 54^{\prime} \mathrm{W}$ & UFG50327 \\
\hline p4 & Córrego Salto Itiquira & $27 / 02 / 2012$ & Formosa & Leste & $15^{\circ} 21^{\prime} \mathrm{S} / 47^{\circ} 27^{\prime} \mathrm{W}$ & UFG50329 \\
\hline p5 & Rio Santa Maria & $27 / 08 / 2012$ & $\begin{array}{l}\text { Alvorada do } \\
\text { Norte }\end{array}$ & Leste & $14^{\circ} 27^{\prime} \mathrm{S} / 46^{\circ} 51^{\prime} \mathrm{W}$ & UFG50330 \\
\hline p6 & Rio João Leite & $27 / 07 / 2012$ & Goiânia & Centro & $16^{\circ} 37^{\prime} \mathrm{S} / 49^{\circ} 14^{\prime} \mathrm{W}$ & UFG50334 \\
\hline p7 & Rio Pirapetinga & $28 / 07 / 2012$ & Caldas Novas & Sudeste & $17^{\circ} 43^{\prime} \mathrm{S} / 48^{\circ} 33^{\prime} \mathrm{W}$ & UFG50335 \\
\hline p8 & Rio após Nova Veneza & $27 / 07 / 2012$ & Nova Veneza & Centro & $16^{\circ} 20^{\prime} \mathrm{S} / 49^{\circ} 25^{\prime} \mathrm{W}$ & UFG50336 \\
\hline p9 & Ribeirão do Bagre & $29 / 07 / 2012$ & Caldas Novas & Sudeste & $17^{\circ} 54^{\prime} \mathrm{S} / 48^{\circ} 39^{\prime} \mathrm{W}$ & UFG50337 \\
\hline
\end{tabular}

Tabela 2 - Frequência de espécies nos ambientes aquáticos lóticos do estado de Goiás ( $\mathrm{F}$-frequência).

Table 2 - Frequency of species in the lotic aquatic environments from Goiás state (F -frequency).

\begin{tabular}{|c|c|c|c|c|c|c|c|c|c|c|}
\hline Táxon & $\mathbf{P 1}$ & $\mathbf{P 2}$ & P3 & $\mathbf{P 4}$ & P5 & P6 & P7 & P8 & P9 & $\mathbf{F}$ \\
\hline Navicula angusta Grunow & $\mathrm{X}$ & & & & & & & & & comum \\
\hline Navicula capitatoradiata Germain & & & & $\mathrm{X}$ & $\mathrm{X}$ & & & $\mathrm{X}$ & $\mathrm{X}$ & comum \\
\hline Navicula insulsa Metzeltin \& Lange-Bertalot & $\mathrm{X}$ & & & & & & $\mathrm{X}$ & & $\mathrm{X}$ & comum \\
\hline Navicula leptostriata Jørgensen & & $\mathrm{X}$ & $\mathrm{X}$ & $\mathrm{X}$ & $\mathrm{X}$ & $\mathrm{X}$ & $\mathrm{X}$ & $\mathrm{X}$ & $\mathrm{X}$ & frequente \\
\hline Navicula lundii Reichardt & & & & & & $\mathrm{X}$ & & & & comum \\
\hline Navicula neomundana Lange-Bertalot, Jarlman \& van de Vijver & & & & & & & $\mathrm{X}$ & & $\mathrm{X}$ & comum \\
\hline Navicula parablis Hohn \& Hellerman & & $\mathrm{X}$ & & $X$ & $\mathrm{X}$ & $\mathrm{X}$ & $\mathrm{X}$ & $\mathrm{X}$ & $\mathrm{X}$ & frequente \\
\hline Navicula radiosa Kützing & & & & & $\mathrm{X}$ & & $\mathrm{X}$ & & $\mathrm{X}$ & comum \\
\hline Navicula rostellata Kützing & & & & $X$ & $\mathrm{X}$ & $\mathrm{X}$ & $X$ & $\mathrm{X}$ & & frequente \\
\hline Navicula symmetrica Patrick & & & & & & $\mathrm{X}$ & $\mathrm{X}$ & $\mathrm{X}$ & $\mathrm{X}$ & comum \\
\hline
\end{tabular}


Navicula angusta Grunow, Verhandlungen der Kaiserlich-Königlichen Zoologisch-Botanischen Gesellschaft in Wein, v. 10, p. 528, pl. 3, fig. 19. 1860.

Fig. 1a-b

Valvas lineares; extremidades sub-rostratas arredondadas; área axial estreita, moderadamente assimétrica; esterno da rafe linear; área central em ângulo agudo com sub-fáscia; rafe filiforme, extremidades proximais dilatadas, extremidades terminais em forma de foice; estrias radiadas na região mediana a convergentes nas extremidades. Comprimento 41,4-48,6 $\mu \mathrm{m}$; Largura 6,4-7,7 $\mu \mathrm{m}$; 13-17 estrias em $10 \mu \mathrm{m}$.

Material examinado: BRASIL. GOIÁS: Alto Paraíso de Goiás, Corredeira dos Ingleses, perifíton, 21.X.2011, Felisberto et al. 40 (UFG50331).

Os espécimes do estudo concordam com os indicados por Lange-Bertalot (2001, página 240 , figuras 1-8) em relação às dimensões e morfologia da valva. Como também confere com as características morfológicas do táxon apresentado em Patrick \& Reimer (1966). N. angusta diferenciase das outras espécies de Navicula por apresentar extremidades sub-rostradas arredondadas. Este táxon foi encontrado em córregos do sul do Brasil, e foi indicadora de ambientes pouco impactados e com valores baixos de nitrogênio, fósforo e turbidez (Schneck et al. 2007).

Distribuição Geográfica para a região CentroOeste: Município de Formosa, Rio Itiquira (Souza \& Oliveira 2007); Município de Nova Roma, Córrego Forquilha (Souza \& Oliveira 2007); Município de São João da Aliança, Ribeirão Faria (Souza \& Oliveira 2007).

Navicula capitatoradiata Germain, Flore des diatomées, p. 188. 1981.

Basiônimo: Navicula cryptocephala var. intermedia Grunow in Van Heurck, Synopsis des diatomées Belgique, pl. 8, fig. 10. $1880 . \quad$ Figs. 1c-e; 3a-d Valvas lanceoladas; extremidades prolongadas capitadas a sub-capitadas; área axial estreita, esterno da rafe estreito; área central levemente elíptica, delimitada por estrias irregulares radiadas (curtas e longas alternadas); rafe filiforme, extremidades proximais dilatadas formando um pequeno gancho e extremidades terminais em forma de foice; estrias fortemente radiadas na região mediana a levemente convergentes nas extremidades. Comprimento 36,4-39,9 $\mu \mathrm{m}$; Largura: 7,4-7,7 $\mu \mathrm{m} ; 15-17$ estrias em $10 \mu \mathrm{m} ; 18$ aréolas em $5 \mu \mathrm{m}$.

Os espécimes de Navicula capitatoradiata apresentaram morfologia e dimensões consistente com o espécime tipo. $N$. capitatoradiata pode ser confundida com Navicula rostellata Kützing pela semelhança do formato valvar e extremidades. No entanto, Navicula rostellata além de apresentar valvas mais elípticas e extremidades menos capitadas, apresenta dimensões valvares maiores (Comprimento 34-50 $\mu \mathrm{m}$; Largura 8-10 $\mu \mathrm{m}$; 11-14 estrias em $10 \mu \mathrm{m}$; Lange-Bertalot 2001). Os espécimes do estudo também foram consistentes com os identificados no trabalho de Leandrini et al. (2002), de amostras perifíticas de rios da região sul do Brasil. Este táxon já foi registrado em uma sub-bacia com grandes níveis de poluição orgânica no sul do país por Heinrich et al. (2014). Apresenta uma grande distribuição na Argentina, encontrada particularmente em ambientes lóticos com temperaturas baixas (Vouilloud et al. 2005). Os espécimes do estudo foram consistentes com as características morfológicas dos espécimes da Argentina, apesar destas apresentarem comprimento e densidade de estrias menor (Comprimento 27-38 $\mu \mathrm{m}$; $12-14$ estrias em $10 \mu \mathrm{m}$; Vouilloud et al. 2005).

Distribuição Geográfica para a região CentroOeste: Brasília, Bacia do Descoberto (Delgado 2006).

Material examinado: BRAZIL. GOIÁS: Formosa, Córrego Salto do Itiquira, perifíton, 27.II.2012, Felisberto, et al. 38 (UFG50329); Flores de Goiás, Rio Santa Maria, perifiton, 27.VIII.2012, Felisberto et al. 39 (UFG50330); Nova Veneza, Rio após Nova Veneza, perifiton, 27.VIII.2012, Felisberto et al. 45 (UFG50336); Caldas Novas, Ribeirão do Bagre, perifiton, 29.VII.2012, Felisberto et al. 46 (UFG50337).

Navicula insulsa Metzeltin \& Lange-Bertalot, Iconographia Diatomologica, v. 5, p. 142, pl. 75, figs. 4-6. 1998.

Figs.1f-g; $3 \mathrm{e}$

Valvas linear-lanceoladas; extremidades prolongadas rostradas; área axial estreita, linear; esterno linear, estreito; área central moderadamente ampla, delimitado pelo encurtamento de estrias (presença de sub-fáscia), ângulo agudo retangular; rafe filiforme, extremidades proximais dilatadas, extremidades terminais em forma de foice; estrias areoladas (aréolas retangulares), estrias radiadas na região mediana, estrias convergentes a paralelas nas extremidades. Comprimento 51,6-54,9 $\mu \mathrm{m}$; Largura $6,5-7,9 ; 15-17$ estrias em $10 \mu \mathrm{m} ; 12$ aréolas em $5 \mu \mathrm{m}$.

A população examinada concorda com o material tipo de Metzeltin \& Lange-Bertalot (1998). Os espécimes inventariados apresentam 


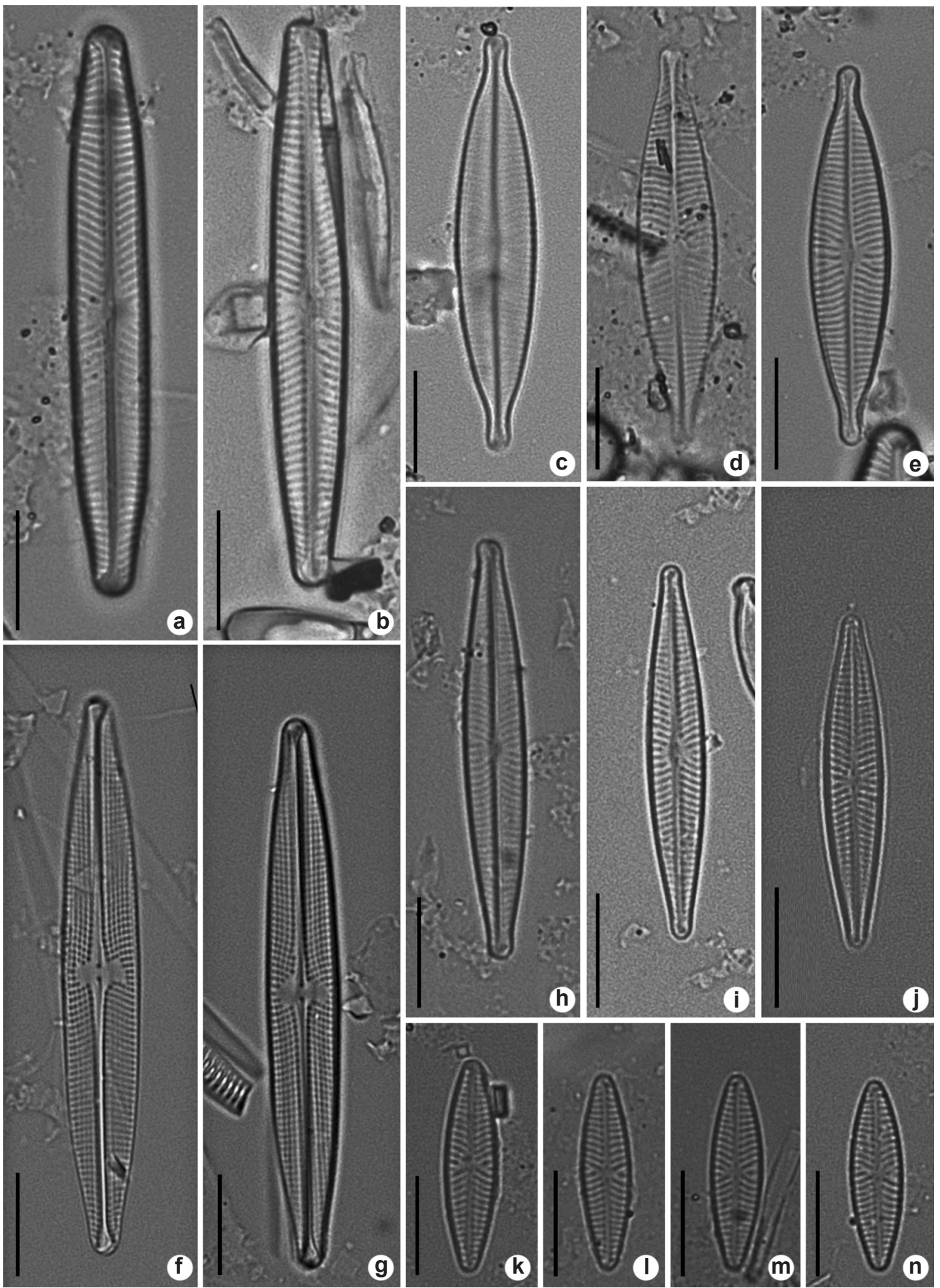

Figura 1 - a-n. Navicula (Naviculaceae) no perifíton de riachos e novas ocorrências para o estado de Goiás - vista valvar em microscopia ótica. a-b. Navicula angusta. c-e. Navicula capitatoradiata. f-g. Navicula insulsa. h-j. Navicula leptostriata. k-n. Navicula lundii. Barra de escala: $10 \mu \mathrm{m}$.

Figure 1 - a-n. Navicula (Naviculaceae) in the periphyton of streams and new occurrences for Goiás state - valvar view on optical microscopy. a-b. Navicula angusta. c-e. Navicula capitatoradiata. f-g. Navicula insulsa. h-j. Navicula leptostriata. k-n. Navicula lundii. Scale bars: $10 \mu \mathrm{m}$. 
formato valvar similar a dos táxons de Navicula herbstiae descrito por Metzeltin \& Lange-Bertalot (1998, página 387, figuras 4-7), mas diferenciamse por Navicula insulsa apresentar valvas mais retangulares, extremidades menos estreitas e estrias menos radiadas. Apesar de também apresentar semelhanças com Navicula coraliana Metzeltin $\&$ Lange-Bertalot, difere por este táxon apresentar menor comprimento valvar e maior linearidade (Metzeltin \& Lange-Bertalot 1998, página, 386, prancha 76). Esta espécie foi registrada em uma represa localizada no sul do Brasil pelo trabalho de Raupp et al. (2006). Apesar do espécime registrado em Raupp et al. (2006) diferir em relação à área central dos espécimes do estado de Goiás, apresenta as mesmas dimensões e demais características morfológicas.

Distribuição Geográfica para a região CentroOeste: Município de Formosa, Rio Paranã (Souza \& Oliveira 2007); Município de Nova Roma, Córrego Forquilha (Souza \& Oliveira 2007); Município de Nova Roma, Rio São Bartolomeu (Souza \& Oliveira 2007).

Material examinado: BRASIL. GOIÁS: Alto Paraíso de Goiás, Corredeira dos Ingleses, perifíton, 21.X.2011, Felisberto et al. 40 (UFG50331); Caldas Novas, Rio Parapetinga, perifíton, 28.VII.2012, Felisberto et al. 44 (UFG50335); Caldas Novas, Ribeirão do Bagre, 29.VII.2012, perifiton, Felisberto et al. 46 (UFG50337).

Navicula leptostriata Jørgensen, Det Kongelige Danske Videnskabernes Selskab, Biologiske Shrifter, v. 5, p. 59, pl. 2, fig. 25. 1948.

Figs. 1h-j; 3f-h

Valvas lanceoladas, estreitas; extremidades estreitas cuneado-arredondadas; área axial estreita, esterno da rafe linear, estreito; área central arredondada, pequena e delimitada por estrias irregulares radiadas, podendo ser assimétrica; rafe filiforme, extremidade proximal levemente defletida e extremidade terminal em forma de foice; estrias radiadas na região mediana a levemente convergentes nas extremidades. Comprimento 21,1-39,3 $\mu \mathrm{m}$; Largura 4,3-5,8 $\mu \mathrm{m} ; 11-18$ estrias em $10 \mu \mathrm{m} ;>13-20$ aréolas em $5 \mu \mathrm{m}$.

Os espécimes do estudo assemelham-se ao tipo apresentado por Jørgensen (1948), no entanto o tipo apresenta extremidades mais proeminentes e maior densidade de estrias como as espécimes registradas em Lange-Bertalot (2001). No entanto, a identificação da espécie foi mantida como $N$. leptostriata devido à semelhança das demais características e pelo valor de densidade de estrias estar de acordo com a variação apresentada por Lange-Bertalot (2001) e Silva et al. (2010) para ambientes europeus e brasileiros, respectivamente. $N$. heimansioides Lange-Bertalot assemelha-se aos espécimes do estudo pelo formato valvar, mas difere por apresentar maior comprimento valvar (Comprimento 30-50 $\mu \mathrm{m}$, Largura 5-6 $\mu \mathrm{m}$, LangeBertalot 2001). N. leptostriata apresenta formato valvar, extremidades e estriação semelhante aos espécimes de Navicula notha Wallace abordado por Lange-Bertalot (2001). No entanto, $N$. notha apresenta área axial estreita e linear, e populações com dimensões menores (Lange-Bertalot 2001, página 317, figuras 16-20). Para o Brasil esta espécie foi encontrada em um reservatório eutrófico na região sul do país (Silva et al. 2010).

Distribuição Geográfica para a região CentroOeste: Brasília, Bacia do Descoberto (Delgado 2006).

Material examinado: BRASIL. GOIÁS: Pirenópolis, Córrego Pirenópolis, perifiton, 16.XII.2011, Felisberto et al. 35 (UFG50326); Pirenópolis, Cachoeira Andorinha, perifíton, 16.XII.2011, Felisberto et al. 36 (UFG50327); Formosa, Córrego Salto do Itiquira, perifíton, 27.II.2012, Felisberto et al. 38 (UFG50329); Alvorada do Norte, Rio Santa Maria, perifíton, 27.VIII.2012, Felisberto et al. 39 (UFG50330); Alto Paraíso de Goiás, Corredeira dos Ingleses, perifíton, 21.X.2011, Felisberto et al. 40 (UFG50331); Goiânia, Rio João Leite, perifíton, 27.VII.2012, Felisberto et al. 43 (UFG50334); Nova Veneza, Rio após Nova Veneza, perifíton, 27.VII.2012, Felisberto et al. 45 (UFG50336); Caldas Novas, Rio Parapetinga, perifíton, 28.VII.2012, Felisberto et al. 44 (UFG50335); Caldas Nova, Ribeirão do Bagre, perifiton, 29.VII.2012, Felisberto et al. 46 (UFG50337).

Navicula lundii Reichardt, Berichte der Bayerischen Botanischen Gessellschaft, p. 180, pl.1, fig. 29-33, pl.3, fig.14. 1985.

Basiônimo: Navicula cryptocephala f. terrestris Lund, New Phytologist, p. 86, text-figs. 9H-9W. 1946.

Figs. 1k-n; $3 \mathrm{i}$

Valvas lanceoladas; extremidades cuneadoarredondadas; área axial estreita; esterno da rafe estreito, linear; área central estreita, levemente irregular; rafe filiforme, extremidades proximais levemente dilatadas, extremidades terminais em forma de foice; estrias radiadas na região mediana, convergentes nas extremidades. Comprimento 18,4-20,4 $\mu \mathrm{m}$; Largura 4,9-6,1 $\mu \mathrm{m} ; 15$ estrias em $10 \mu \mathrm{m} ; 17$ aréolas em $5 \mu \mathrm{m}$.

Lange-Bertalot (2001) comenta sobre a dificuldade de identificação deste táxon devido à sua semelhança com certas espécies como Navicula 

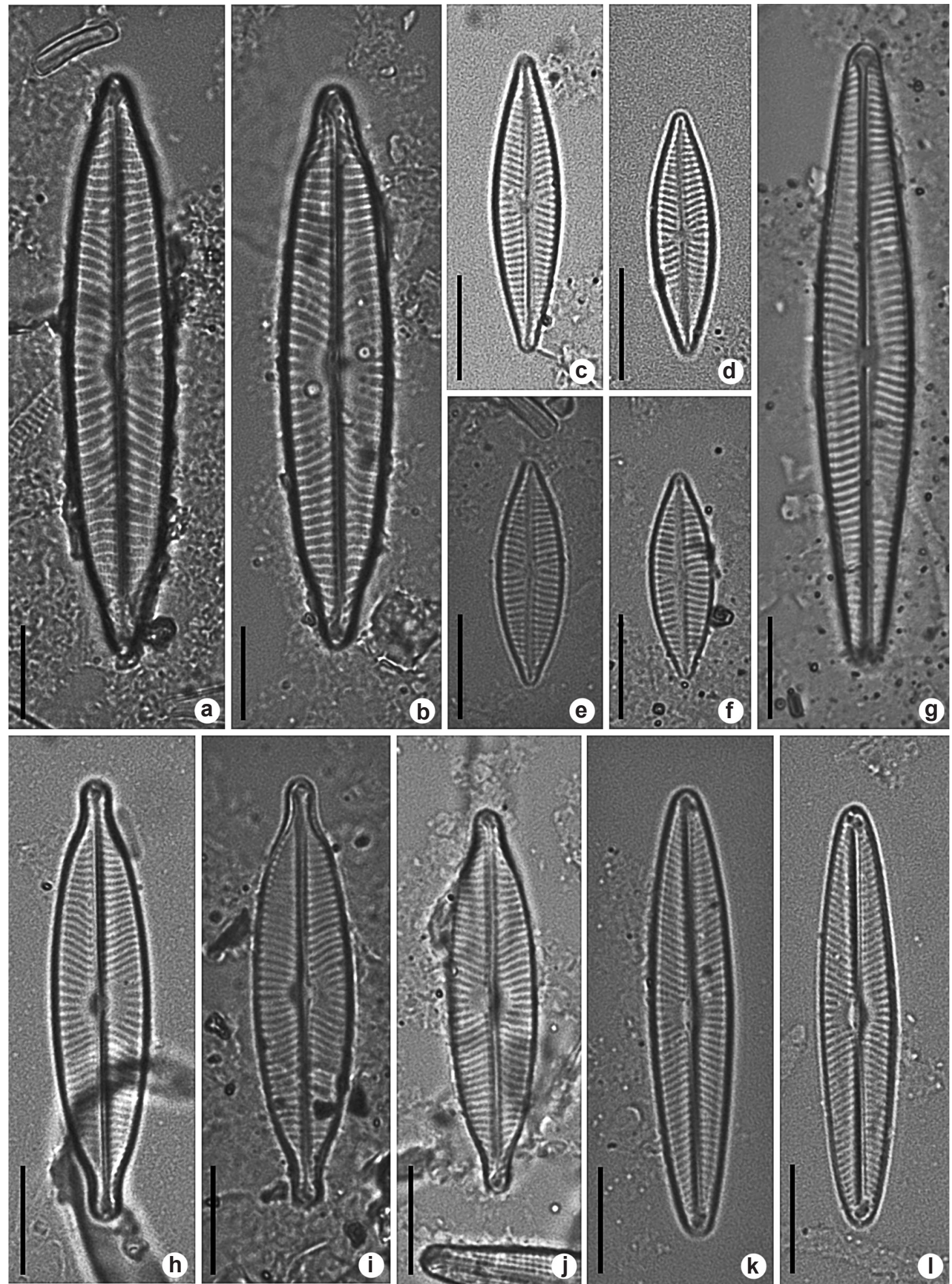

Figura 2 - a-1. Navicula (Naviculaceae) no perifíton de riachos e novas ocorrências para o estado de Goiás - vista valvar em microscopia ótica. a-b. Navicula neomundana. c-f. Navicula parablis. g. Navicula radiosa. h-j. Navicula rostellata. k-1. Navicula symmetrica. Barra de escala: $10 \mu \mathrm{m}$.

Figure 2 -a-1. Navicula (Naviculaceae) in the periphyton of streams and new occurrences for Goiás state - valvar view on optical microscopy a. Navicula neomundana. b-d. Navicula parablis. e. Navicula radiosa. f-g. Navicula rostellata. h-j. Navicula symmetrica. Scale bars: $10 \mu \mathrm{m}$. 
cryptocephala Kützing e Navicula exilis Kützing, e argumenta ainda que todas as características morfológicas devem ser consideradas para a diferenciação destes táxons. $N$. lundii difere de $N$. cryptocephala principalmente por este último táxon apresentar comprimento e largura maiores. $N$. exilis difere-se de $N$. lundii por nitidamente apresentar extremidades obtusas. Portanto, os espécimes do estudo foram mantidos como $N$. lundii. Este táxon também assemelha-se morfologicamente a Navicula veneta Kützing, por apresentar dimensões e características morfológicas similares (Comprimento 13-30 $\mu \mathrm{m}$; Largura 5-6 $\mu \mathrm{m}$; Lange-Bertalot 2001). No entanto, Navicula veneta apresenta moderadamente extremidades sub-capitadas e área axial com estrias simétricas e pouco radiadas.

Distribuição Geográfica para a região CentroOeste: Brasília, Bacia do Descoberto (Delgado 2006); Município Flores de Goiás, Rio dos Macacos (Souza \& Oliveira 2007); Município de Formosa, Córrego Água Fria (Souza \& Oliveira 2007); Município de Formosa, Rio Jataí (Souza \& Oliveira 2007); Município de Formosa, Rio Mato Grosso (Souza \& Oliveira 2007); Município de Nova Roma, Córrego Forquilha (Souza \& Oliveira 2007); Município de São João da Aliança, Ribeirão Faria (Souza \& Oliveira 2007); Município de São João de Aliança, Ribeirão Extrema (Souza \& Oliveira 2007). Material examinado: BRASIL. GOIÁS: Goiânia, Rio João Leite, perifíton, 27.VII.2012, Felisberto et al. 43 (UFG50334).

Navicula neomundana (Lange-Bertalot \& Rumrich) Lange-Bertalot, Jarlman \& van de Vijver in van de Vijver \& Lange-Bertalot, Diatom Research, p. 424. 2009.

Basiônimo: Navicula viridulacalcis subsp. neomundana Lange-Bertalot \& Rumrich in Rumrich, Lange-Bertalot, \& Rumrich, Iconographia Diatomologica, v. 9, 175, pl. 37, figs. 5-8, pl. 38, figs. 1-4. $2000 . \quad$ Figs. 2a-b

Valvas lineares a linear-lanceoladas; extremidades prolongadas cuneado-sub-rostradas; área axial estreita, linear; esterno linear, estreito; área central ampla, circular, assimétrico, com espaçamento de sílica evidente; rafe filiforme, extremidade proximal defletida para o mesmo lado e extremidades terminais em forma de foice; estrias areoladas, radiadas na região mediana e convergentes nas extremidades. Comprimento 55,2-71,4 $\mu \mathrm{m}$; Largura: 10,2-10,8 $\mu \mathrm{m}$; 9-10 estrias em $10 \mu \mathrm{m}$.
Navicula neomundana difere de outras espécies do gênero Navicula por apresentar valvas lineares a linear-lanceoladas com extremidades prolongadas cuneadas-sub-rostradas (Vijver \& Lange-Bertalot 2009). Os espécimes deste estudo foram identificados conforme os registrados em trabalhos brasileiros de Bartozek et al. (2013) e Bertolli et al. (2010). Os espécimes estudados apresentaram semelhanças morfológicas (formato valvar, extremidades, estrias e área axial) com os indivíduos de $N$. amphiceropsis Lange-Bertalot \& Rumrich apontados em Metzeltin et al. (2005, página 327 , figuras 1-16). No entanto os espécimes foram mantidos como $N$. neomundana, pois o espécime tipo de $N$. amphiceropsis em Rumrich et al. (2000) difere dos demonstrados em Metzeltin et al. (2005).

Distribuição Geográfica para a região CentroOeste: primeiro registro.

Material examinado: BRASIL. GOIÁS: Caldas Novas, Rio Parapetinga, perifiton, 28.VII.2012, Felisberto et al. 44 (UFG50335); Caldas Nova, Ribeirão do Bagre, perifíton, 29.VII.012, Felisberto et al. 46 (UFG50337);

Navicula parablis Hohn \& Hellerman, Transactions of the American Microscopical Society, v. 82, p. 307, pl. 3, fig. 17. $1963 . \quad$ Figs. 2c-f; 3j-k

Valvas lanceoladas; extremidades cuneadas; área axial estreita; esterno da rafe estreito e área central estreita delimitada por estrias irregulares, levemente assimétrica; rafe filiforme, extremidade proximal dilatada e extremidade terminal em forma de foice; estrias radiadas na região mediana e convergentes nas extremidades. Comprimento 18,6-39,9 $\mu \mathrm{m}$; Largura 5-7 $\mu \mathrm{m} ; 14-18$ estrias em $10 \mu \mathrm{m} ; 16-18$ aréolas em $5 \mu \mathrm{m}$.

A população examinada confere com os espécimes de Navicula parablis indicados em Rumrich et al. (2000). Os espécimes do estudo assemelham-se em relação ao formato valvar a Navicula quasidisjuncta Lange-Bertalot \& Rumrich de Rumrich et al. (2000) e Navicula cryptotenella Lange-Bertalot. No entanto, diferemse pelo comprimento, largura e densidade de estrias (Rumrich et al. 2000; Lange-Bertalot 2001). $N$. cryptotenella, diferencia-se de $N$. parablis por este apresentar extremidades cuneadas e maior comprimento valvar (comprimento $12-40$ $\mu \mathrm{m}$, Lange-Bertalot 2001). Enquanto que $N$. quasidisjuncta diferencia-se de $N$. parablis por apresentas menor comprimento valvar (comprimento 20-27 $\mu \mathrm{m}$, Rumrich et al. 2000).

Distribuição Geográfica para a região CentroOeste: primeiro registro. 

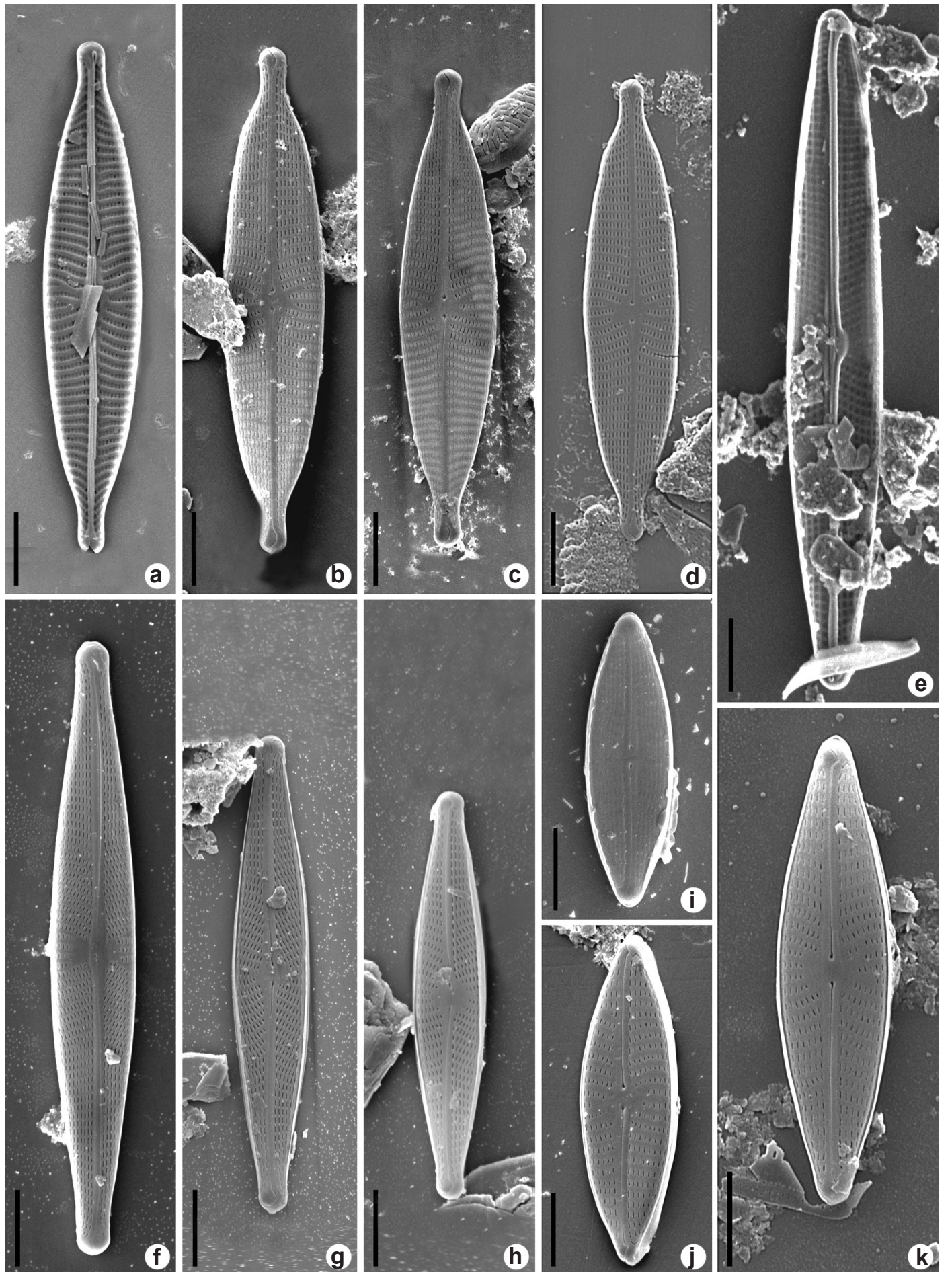

Figura 3 - a-k. Navicula (Naviculaceae) no perifíton de riachos e novas ocorrências para o estado de Goiás - vista valvar em microscopia eletrônica de varredura. a-d. Navicula capitatoradiata - a. vista interna; b-d. vista externa. e. Navicula insulsa - vista interna. f-h. Navicula leptostriata - vista externa. i. Navicula lundii - vista externa. j-k. Navicula parablis - vista externa. Barra de escala: $5 \mu \mathrm{m}$.

Figure 3 - a-k. Navicula (Naviculaceae) in the periphyton of streams and new occurrences for Goiás state - valvar view on scanning electronic microscopy. a-d. Navicula capitatoradiata - a. internal view; b-d. external view. e. Navicula insulsa - internal view. f-h. Navicula leptostriata - external view. i. Navicula lundii - external view. j-k. Navicula parablis - external view. Scale bars: $5 \mu \mathrm{m}$. 
Material examinado: BRASIL. GOIÁS: Pirenópolis, Córrego Pirenópolis, perifíton, 16.XII.2011, Felisberto et al. 36 (UFG50327); Formosa, Córrego Salto do Itiquira, perifíton, 27.II.2012, Felisberto, et al. 38 (UFG50329); Flores de Goiás, Rio Santa Maria, perifíton, 27.VIII.2012, Felisberto et al. 39 (UFG50330); Goiânia, Rio João Leite, perifíton, 27.VII.2012, Felisberto et al. 43 (UFG50334); Nova Veneza, Rio após Nova Veneza, perifíton, 27.VII.2012, Felisberto et al. 45 (UFG50336); Caldas Novas, Rio Parapetinga, perifíton, 28.VII.2012, Felisberto et al. 44 (UFG50335); Caldas Nova, Ribeirão do Bagre, perifíton, 29.VII.2012, Felisberto et al. 46 (UFG50337).

Navicula radiosa Kützing, Bacillarien, p. 91, pl.4, fig. 23. 1844.

Fig. $2 \mathrm{~g}$

Valvas lanceoladas; extremidades prolongadas, cuneado-arredondadas; área axial estreita; esterno da rafe estreito, linear; área central rômbica; rafe filiforme, parte terminal levemente defletida para o mesmo lado em forma de foice; estrias radiadas a paralelas na região mediana e convergentes nas extremidades. Comprimento $55,5-65,9 \mu \mathrm{m}$; Largura 8,2-10,6 $\mu \mathrm{m} ; 12$ estrias em $10 \mu \mathrm{m}$.

A população examinada concorda com os espécimes apontados em Rumrich et al. (2000), Lange-Bertalot (2001) e Taylor et al. (2007). Esta espécie foi registrada em ambientes lóticos brasileiros por Wengrat et al. (2008) e Leandrini et al. (2002), sendo que os espécimes deste último trabalho apresentam dimensões e características morfológicas que conferem com os indivíduos do presente estudo. Apesar da semelhança morfológica de $N$. radiosa e Navicula lohmanni Lange-Bertalot \& Rumrich, $N$. radiosa apresenta dimensões maiores e área axial distinta (Lange-Bertalot 2000).

Distribuição Geográfica para a região CentroOeste: Município de Itajá, Lagoa Santa (Contin \& Oliveira, 1993); Brasília, Bacia do Descoberto (Delgado 2006); Município de Formosa, Córrego Água Fria (Souza \& Oliveira 2007); Município de Formosa, Rio Jataí (Souza \& Oliveira 2007); Município de Formosa, Rio Itiquira (Souza \& Oliveira 2007); Município de Formosa, Rio Salobro (Souza \& Oliveira 2007); Município de Formosa, Rio Crixás (Souza \& Oliveira 2007); Município de Formosa, Rio Mato Grosso (Souza \& Oliveira 2007); Município de Formosa, Rio Paraná (Souza \& Oliveira 2007); Município de Formosa, Rio Cangalha (Souza \& Oliveira 2007); Município de Iacira, Estação 1 (Souza \& Oliveira 2007); Município de Iacira, Rio São Mateus (Souza \& Oliveira 2007); Município de Iacira, Rio São
Paranã (Souza \& Oliveira 2007); Município de Nova Roma, Córrego Morcego (Souza \& Oliveira 2007); Município de Nova Roma, Córrego Forquilha (Souza \& Oliveira 2007); Município de Nova Roma, Rio São Bartolomeu (Souza \& Oliveira 2007); Município de São João de Aliança, Ribeirão Extrema (Souza \& Oliveira 2007).

Material examinado: BRAZIL. GOIÁS: Flores de Goiás, Rio Santa Maria, perifíton, 27.VIII.2012, Felisberto et al. 39 (UFG50330); Caldas Novas, Rio Parapetinga, perifiton, 28.VII.2012, Felisberto et al. 44 (UFG50335); Caldas Novas, Ribeirão do Bagre, 29.VII.2012, perifiton, Felisberto et al. 46 (UFG50337);

Navicula rostellata Kützing, Bacillarien, p. 95, pl. 3, fig. 65. $1844 . \quad$ Figs. 2h-j; 4a-c

Valvas lanceoladas-elípticas; capitadas a sub-capitadas; área axial estreita; esterno da rafe estreito; área central elíptica e levemente assimétrica; rafe filiforme, extremidade proximal dilatada, defletidas para o mesmo lado, extremidade terminal em forma de foice; estrias radiadas a convergentes na região mediana e paralelas nas extremidades. Comprimento 31,4-40,6 $\mu \mathrm{m}$; Largura 7,4-9,3 $\mu \mathrm{m} ; 13-15$ estrias em $10 \mu \mathrm{m}$; 18-20 aréolas em $5 \mu \mathrm{m}$.

Navicula rostellata apresenta semelhanças morfológicas (formato valvar) com Navicula viridula Ehrenberg. Devido à estas semelhanças muitos autores citam este táxon de acordo com seu basiônimo $N$. virudula var. rostellata, como nos trabalhos brasileiros de Leandrini et al. (2002) e Oliveira et al. (2001). No entanto, Lange-Bertalot (2001) difere Navicula rostellata de $N$. viridula, por esta apresentar forma valvar e extremidades mais alongadas e maior largura valvar (Comprimento 40-100 $\mu \mathrm{m}$; Largura 10-15 $\mu \mathrm{m}$, Lange-Bertalot 2001). Esta espécie também foi registrada para o Brasil pelos trabalhos de Bartozek et al. (2013), Bertolli et al. (2010) e Lobo et al. (2004), nos quais os espécimes encontrados conferem com os do estado de Goiás.

Distribuição Geográfica para a região Centro-Oeste: Brasília, Bacia do Descoberto (Delgado 2006); Município Flores de Goiás, Rio dos Macacos (Souza \& Oliveira 2007); Município de Formosa, Rio Jataí (Souza \& Oliveira 2007); Município de Formosa, Rio Paranã (Souza \& Oliveira 2007); Município de Formosa, Rio Salobro (Souza \& Oliveira 2007); Município de Formosa, Rio Crixás (Souza \& Oliveira 2007); Município de Formosa, Rio Mato Grosso (Souza \& Oliveira 2007); Município de Formosa, Rio Paraná (Souza 

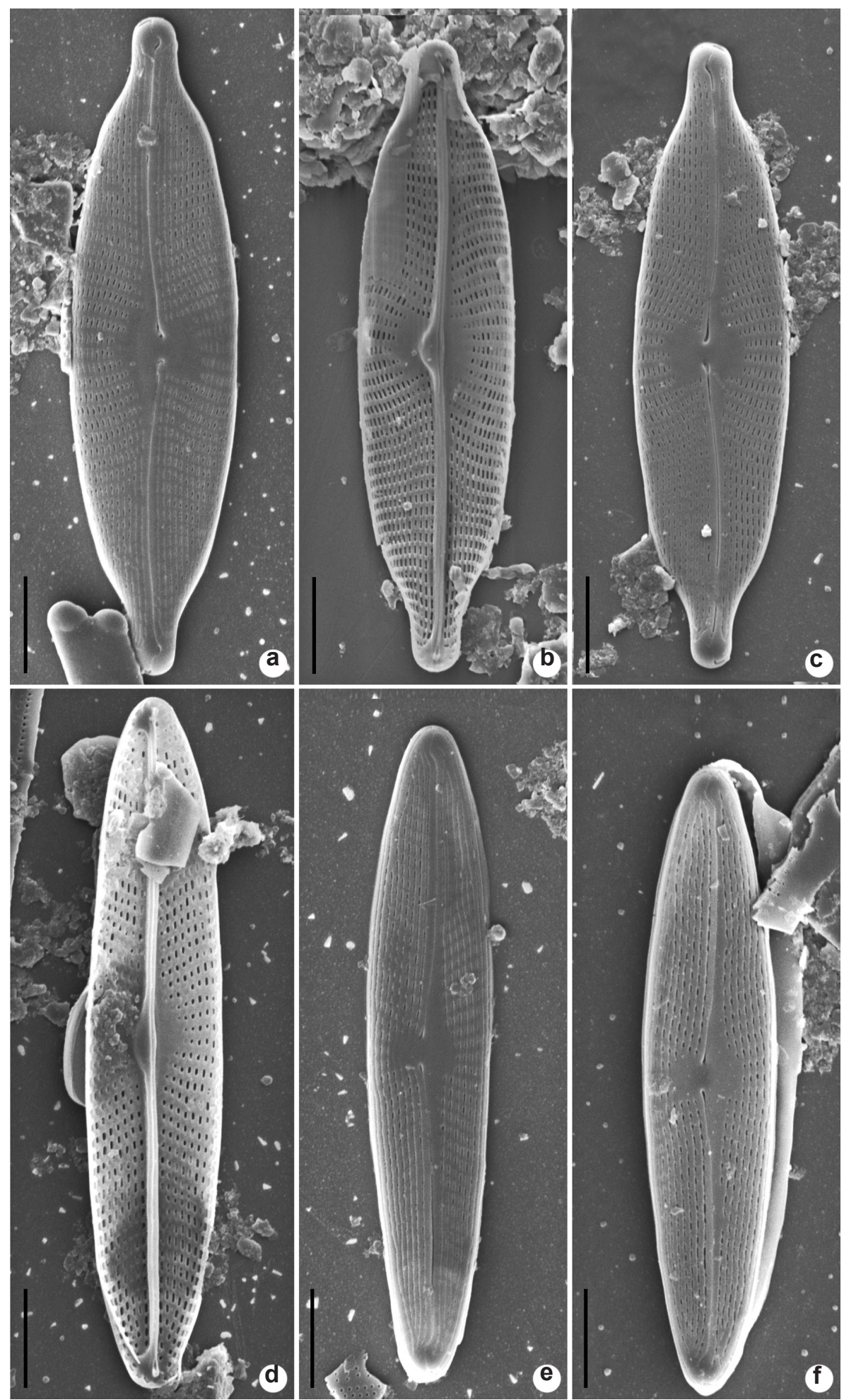

Figura 4 - a-k. Navicula (Naviculaceae) no perifíton de riachos e novas ocorrências para o estado de Goiás - vista valvar em microscopia eletrônica de varredura. a-c. Navicula rostellata - a,c. vista externa; b. vista interna. d-f. Navicula symmetrica - d. vista interna; e-f. vista externa). Barra de escala: $5 \mu \mathrm{m}$.

Figure 4-a-k. Navicula (Naviculaceae) in the periphyton of streams and new occurrences for Goiás state-valvarview on scanning electronic microscopy. a-c. Navicula rostellata - a,c. external view; b. internal view. d-f. Navicula symmetrica - d. internal view; e-f. external view). Scale bars: $5 \mu \mathrm{m}$. 
\& Oliveira 2007); Município de Formosa, Rio Cangalha (Souza \& Oliveira 2007); Município de Iacira, Estação 1 (Souza \& Oliveira 2007); Município de Iacira, Estação 1 (Souza \& Oliveira 2007); Município de Iacira, Rio São Mateus (Souza \& Oliveira 2007); Município de Nova Roma, Córrego Morcego (Souza \& Oliveira 2007); Município de Nova Roma, Rio São Bartolomeu (Souza \& Oliveira 2007); Município de São João de Aliança, Ribeirão Faria (Souza \& Oliveira 2007); Município de São João de Aliança, Ribeirão Piripiri (Souza \& Oliveira 2007); Município de São João de Aliança, Rio São Pedro (Souza \& Oliveira 2007); Município de Vila Boa, Córrego Extrema (Souza \& Oliveira 2007).

Material examinado: BRASIL. GOIÁS: Formosa, Córrego Salto do Itiquira, perifíton, 27.II.2012, Felisberto et al. 38 (UFG50329); Flores de Goiás, Rio Santa Maria, perifiton, 27.VIII.2012, Felisberto et al. 39 (UFG50330); Goiânia, Rio João Leite, perifíton, 27.VII.2012, Felisberto et al. 43 (UFG50334); Nova Veneza, Rio após Nova Veneza, perifíton, 27.VIII.2012, Felisberto et al. 45 (UFG50336); Caldas Novas, Rio Parapetinga, 28.VII.2012, perifíton, Felisberto et al. 44 (UFG50335).

Navicula symmetrica Patrick, Boletim do Museu Nacional, p. 5, fig. 6. $1944 . \quad$ Figs. 2k-1; 4d-f Valvas linear-elípticas; extremidades cuneado-arredondadas; área axial estreita; esterno assimétrico principalmente na região mediana; área central semicircular, assimétrica; rafe filiforme, parte proximal defletida para o mesmo lado, parte terminal em forma de foice; estrias radiadas ao longo da valva. Comprimento 33,4-41,5 $\mu \mathrm{m}$; Largura 6,2-7,5 $\mu \mathrm{m} ; 16$ estrias em $10 \mu \mathrm{m} ; 14-18$ aréolas em $5 \mu \mathrm{m}$.

Os espécimes de Navicula symmetrica apresentam características morfológicas semelhantes com Navicula schroeteri Meister. Ambas espécies apresentam forma valvar lanceolada, área central circular assimétrica e estrias radiadas (Lange-Bertalot 2001). LangeBertalot (2001) discute que esses dois táxons são inconfundíveis com outras espécies devido às suas peculiaridades taxonômicas. Este mesmo deixa claro a necessidade de mais estudos de biologia populacional para determinar se são duas espécies ou dois táxons intraespecíficos. No entanto, a população do estudo foi mantida como $N$. symmetrica devido à menor densidade de estrias em Navicula schroeteri (12-15 estrias em $10 \mu \mathrm{m}$ em Lange-Bertalot 2001 e 11-12 estrias em $10 \mu \mathrm{m}$ em Metzeltin \& Lange-Bertalot 2007). Os espécimes encontrados no estado de Goiás conferiram com os registrados por trabalhos brasileiros do sul do país, um realizado em um ambiente lêntico (Torgan et al. 2009) e outro em um ambiente lótico (Heinrich et al. 2014).

Distribuição Geográfica para a região CentroOeste: primeiro registro.

Material examinado: BRASIL. GOIÁS: Goiânia, Rio João Leite, perifíton, Felisberto et al. 43 (UFG50334); Nova Veneza. Rio após Nova Veneza, perifíton, 27.VIII.2012, Felisberto et al. 45 (UFG50336); Caldas Novas, Rio Parapetinga, perifíton, 28.VII.2012, Felisberto et al. 44 (UFG50335); Caldas Nova, Ribeirão do Bagre, perifíton, 29.VII.2012, Felisberto et al. 46 (UFG50337).

\section{Agradecimentos}

O presente trabalho foi realizado com apoio da Coordenação de Aperfeiçoamento de Pessoal de Nível Superior - Brasil (CAPES). Agradecemos a instituição, a bolsa de Mestrado concedida a Daiane T. Ruwer e a Alline A. França, e a bolsa de PRODOC concedida a S.A. Felisberto (in memorian); ao Laboratório Multiusuário de Microscopia de Alta Resolução (LabMic) do Instituto de Física da Universidade Federal de Goiás, o uso do microscópio eletrônico de varredura; ao Laboratório de Análise e Gerenciamento Ambiental de Recursos Hídricos, sob coordenação dos professores Dr. Leandro Gonçalves de Oliveira e Dra. Ina de Souza Nogueira, por concederem o equipamento e espaço físico para a análise do material. Agradecemos a Dr. Liliana Rodrigues, o auxílio nas correções do manuscrito.

\section{Referências}

Bartozek ECR, Ludwig TAV, Tremarin PI, Nardelli MS, Bueno NC \& Rocha ACR (2013) Diatoms (Bacillariophyceae) of Iguaçu National Park, Foz do Iguaçu, Brazil. Acta Botanica Brasilica 27: 108-123.

Bere T \& Tundisi JG (2010) Biological monitoring of lotic ecosystems: the role of diatoms. Brazilian Journal of Biology 70: 493-502.

Bertolli LM, Tremarin PI \& Ludwig TAV (2010) Diatomáceas perifíticas em Polygonum hydropiperoides Michaux, reservatório do Passaúna, Região Metropolitana de Curitiba, Paraná, Brasil. Acta Botanica Brasilica 24: 1065-1081.

Bicudo CEM \& Menezes M (2017) Gêneros de algas de águas continentais do Brasil (Chave de identificação e descrições). $3^{\mathrm{a}}$ ed. Rima, São Carlos. 552p.

Contin LF \& Oliveira RJM (1993) Diatomáceas. In: Campos IFP (coord.) Flora do estado de Goiás. Criptógamos. Vol. 2. ABEU/CEGRAF-UFG. Pp. 35. 
Cox EJ (1999) Studies on the diatom genus Navicula Bory. VIII. Variation in valve morphology in relation to the generic diagnosis based on Navicula tripunctata (OF, Muller) Bory. Diatom Research 14: 207-237.

Delgado SM (2006) Levantamento Florístico das Naviculales (Bacillariophyceae) perifíticas do rio Descoberto, Goiás e Distrito Federal. Dissertação de Mestrado. Universidade de Brasília, Brasília. 177p.

Flora do Brasil, 2020. (em construção). Jardim Botânico do Rio de Janeiro. Disponível em: $<$ http:// floradobrasil.jbrj.gov.br/reflora/floradobrasil/ FB98297>. Acesso em: 13 Mar. 2019

Guille A (1970) Benthic bionomy of continental shelf of the French Catalane Coast. II. Benthic communities of the macrofauna. Vie et Milieu 21: 149-280.

Heinrich CG, Leal VL, Schuch M, Düpont A \& Lobo EA (2014) Epilithic diatoms in headwater areas of the hydrographical subbasin of the Andreas Stream, RS, Brazil, and their relation with eutrophication processes. Acta Limnologica Brasiliensia 26: 347-355.

Hustedt F (1959) Die Kieselalgen Deutschlands, Osterreichs und der Schweiz, 2. Teil. In: Rabenhorst L (ed.) Kryptogamen-Flora von Deutschlands, Osterreichs und der Schweiz. Band VII(2), reprint 1977. Otto Koeltz Science Publishers, Koenigstein. $845 \mathrm{p}$.

Kulikovskiy MS (2008) The species composition and distribution of diatoms in sphagnum bogs of European Russia: ecosystems of the Volga upland. Inland Water Biology 1: 347-355.

Lange-Bertalot H (2000) Transfer to the generic rank of Decussata Patrick as a subgenus of Navicula Bory sensu lato. Iconographica Diatomologica 9: 670-673.

Lange-Bertalot H (2001) Navicula sensu stricto 10 genera separated from Navicua sensu lato Frustulia. Diatoms os Europe. Vol. 2. Gantner Verlag, Germany. 526p.

Leandrini JA, Moreira Filho H \& Rodrigues L (2002) Espécies perifíticas de Navicula Bory de dois sistemas lóticos do município de Maringá, estado do Paraná, Brasil. Hoehnea 29: 49-56.

Lobo EA, Callegaro VL, Hermany G, Bes D, Wetzel CE \& Oliveira MA. (2004) Use of epilithic diatoms as bioindicator from lotic systems in southern Brazil, with special emphasis on eutrophication. Acta Limnologica Brasiliensia 16: 25-40.

Mann DG (1989) The diatom genus Sellaphora: separation from Navicula. British Phycological Journal 24: 1-20.

Metzeltin D \& Lange-Bertalot H (1998) Tropical diatoms of South America. Iconographia Diatomologica $(H$. Lange-Bertalot, ed.). Vol. 5. Koeltz Scientifc Books, Stuttgart. 286p.
Metzeltin D, Lange-Bertalot H \& García-Rodriguez F (2005) Diatoms of Uruguay. In: Lange-Bertalot H (ed.) Iconographia diatomologica. Vol. 15. Koeltz Scientific Books, Königstein. 737p.

Metzeltin D \& Lange-Bertalot H (2007) Tropical Diatoms of South America II. In: Lange-Bertalot H (ed.) Iconographia diatomologica. Vol. 18. Koeltz Scientific Books, Königstein. 877p.

Moreira-Filho H \& Valente-Moreira IM (1981) Avaliação taxonômica e ecológica das diatomáceas (Bacillariophyceae) epífitas em algas pluricelulares obtidas nos litorais dos estados dos estados do Paraná, Santa Catarina e São Paulo. Boletim Museu Botânico Municipal de Curitiba 47: 1-17.

Oliveira MA, Torgan LC, Lobo EA \& Scharzbold A (2001) Association of periphytic diatom species of artificial substrate in lotic environments in the Arroio Sampaio Basin, RS, Brazil: relationship with abiotic variables. Brazilian Journal of Biology 61: 523-540.

Pan Y, Stenvenson RJ, Hill BH, Herlihy AT \& Collins GB (1996) Using diatoms as indicators of ecological conditions in lotic systems: a regional assessment. Journal of North American Bethological Society 15: 481-495.

Patrick R (1959) New subgenera and two new species of the genus Navicula (Bacillariophyceae). Notulae, Naturae 324: 1-1.

Patrick R \& Reimer CW (1966) The diatoms of United States. Vol I. The Academy of Natural Sciences of Philadelphia, Philadelphia. 688p.

PNRH - Plano Nacional de Recursos Hídricos (2006) GAP/DPE/SRH/MMA, Brasília. 132p.

Raupp SV, Torgan LC \& Baptista LRM (2006) Composição e variação temporal de diatomáceas (Bacillariophyta) no plâncton da represa de Canastra, sul do Brasil. Iheringia, Série Botânica 61: 105-134.

Rodrigues L (1992) Diatomoflorula do Rio Tubarao, estado de Santa Catarina, Brasil: II. Naviculaceae. Insula 21: 51-97.

Round FE, Crawford RM \& Mann DG (1990) The diatoms: biology and morphology of the genera. Cambridge University Press, Cambridge. 747p.

Rumrich U, Lange-Bertalot H \& Rumrich M (2000) Diatoms of the Andes from Venezuela to Patagonia/ Tierra del Fuego and two additional contributions. In: Lange-Bertalot $\mathrm{H}$ (ed.) Iconographia diatomologica. Vol. 9. Koeltz Scientific Books, Königstein. 673p.

Schneck F, Torgan LC \& Schwarzbold A (2007) Epilithic diatom community in high altitude stream impacted by fi shfarming in southern Brazil. Acta Limnologica Brasiliensia 19: 341-355.

Silva AM, Ludwig TAV, Tremarin PI \& Vercellino IS (2010) Diatomáceas perifíticas em um sistema eutrófico brasileiro (Reservatório do Iraí, estado 
do Paraná). Acta Botanica Brasilica 24: 997-1016.

Silva WJ, Nogueira IS \& Souza MGM (2011) Catálogo de Diatomáceas da Rsegião Centro-Oeste brasileira. Iheringia, Série Botânica 66: 61-86.

Simonsen R (1974) The diatom plankton of the Indian Ocean Expedition of R/V "Meteor", 1964-65 "Meteor" Forschungsergbnisse. Reihe D-Biologie 19: 1-66.

Souza MGM \& Oliveira RIR (2007) Levantamento da diatomoflórula epilítica da bacia do rio Paranã, Goiás, Brasil. In: Martins-Silva MJ (org.) Inventário da biota aquática com vistas a conservação e utilização sustentável do bioma Cerrado (Serra e Vale do rio Paranã). MMA 1: 72-92.

Taylor JC, Harding WR \& Archibald CGM (2007) A methods manual for the collection, preparation and analysis of diatom samples. Water Research Commission, Pretoria. 49p.

Torgan LC, Salomoni SE \& Bicca AB (2009) Diatomáceas sobre Limnoperma fortunei (Dunker), molusco introduzido no Lago Guaíba, Sul do Brasil. Revista
Brasileira de Botânica 32: 23-31.

Torgan LC, Donadel L \& Silva JG (2010) A transferência de Navicula sovereignae Hustedt para o gênero Placoneis Mereschkowsky (Bacillariophyta). Iheringia, Série Botânica 65: 107-114.

Vijver BV \& Lange-Bertalot H (2009) New and interesting Navicula taxa from Western and Northern Europe. Diatom Research 24: 415-429.

Vouilloud AA, Sala SE, Sabbatini MR (2005) Diatomeas perifíticas de la cuenca del Río Sauce Grande (Buenos Aires, Argentina). Iheringia, Série Botânica 60: 77-89.

Wengrat S, Tavares B, Silva AM \& Aquino NF (2007) Bacillariophyceae do Rio São Francisco Falso, município de Santa Helena, Paraná, Brasil: Navicula. (Nota Científica). Revista Brasileira de Biociências 5: 996-998.

Wengrat S, Tavares B \& Silva AM (2008) Gêneros de Diatomáceas (Ochrophyta) Dulciaquícolas do oeste do estado do Paraná, Brasil. (Nota Científica). Revista Brasileira de Biociências 6: 5-7. 